\title{
NOBLE LIQUID CALORIMETRY AT THE LHC AND PROSPECTS OF ITS APPLICATION IN FUTURE COLLIDER EXPERIMENTS
}

^ Martin Aleksa - EP Department, CERN, Geneva - DOI: https://doi.org/10.1051/epn/2021306

Calorimetry is an important measurement technique in experimental particle physics. Although calorimeters based on liquefied noble gases were first proposed $\mathbf{5 0}$ years ago, they continue to play an important role in modern particle physics and have substantially contributed to the discovery of the Higgs boson at the Large Hadron Collider (LHC) at CERN in 2012.

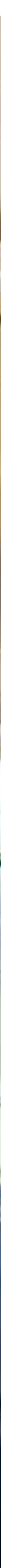


alorimetry refers to the absorption of a particle and the transformation of its energy into a measurable signal related to the energy of the particle [1]. A calorimetric measurement requires that the particle is completely absorbed and is thus no longer available for subsequent measurements. Since the energy of the incident particle is usually much higher than the threshold of inelastic reactions between the particle and the detector medium, the energy loss will produce a cascade of lower energy particles, whose number is proportional to the incident energy. Charged particles in the shower ultimately loose their energy through elementary processes, mainly by ionization and atomic level excitation.

\section{Electromagnetic calorimeters}

In electromagnetic calorimeters, specialized in the measurement of photons and electrons, the shower development is characterised by the radiation length $\mathrm{X}_{0}$ which is defined as the mean distance in which electrons lose all but $1 / \mathrm{e}$ of their initial energy by radiation.

To fit into limited space, electromagnetic calorimeters need to be made of dense materials with a short radiation length. Sufficient longitudinal shower containment for typical particle energies requires a depth of above $20 \mathrm{X}_{0}$. This can be achieved either by homogeneous calorimeters, usually consisting of very dense scintillating crystals (e.g. $\mathrm{PbWO}_{4}, \mathrm{X}_{0}=8.9 \mathrm{~mm}, \mathrm{LYSO}, \mathrm{CsI}, \ldots$ ) or sampling calorimeters in which high-Z absorbers (e.g. $\mathrm{Pb}, \mathrm{X}_{0}=5.6 \mathrm{~mm}$ or $\mathrm{W}, \mathrm{X}_{0}=3.5 \mathrm{~mm}$ ) are stacked with active medium and electrodes interleaved. The left picture of Figure 1 illustrates this principle. Compared to homogeneous calorimeters consisting exclusively of active medium, sampling calorimeters usually exhibit worse energy resolution, since only a statistically fluctuating fraction of the shower leads to energy deposits in the active medium.

Calorimeters using liquefied noble gases (noble liquids) are based on the measurement of the ionization charge produced inside the liquid. As shown in the right sketch of figure 1, the charges move in an applied electric field, inducing a current in readout electrodes proportional to the liberated charge and hence to the energy deposited by the showering particle.

\section{Noble liquid calorimetry}

This technique was introduced in the early 1970s [2] using liquefied argon (LAr) as the active material. Other suitable materials are the heavier noble liquids ( $\mathrm{LKr}, \mathrm{LXe}$ ), however, due to their much higher price and limited availability, they are less popular for large calorimeter systems in collider experiments.

Noble liquid ionization calorimeters offer a number of attractive advantages, especially for collider experiments. They are characterized by intrinsic stability and excellent
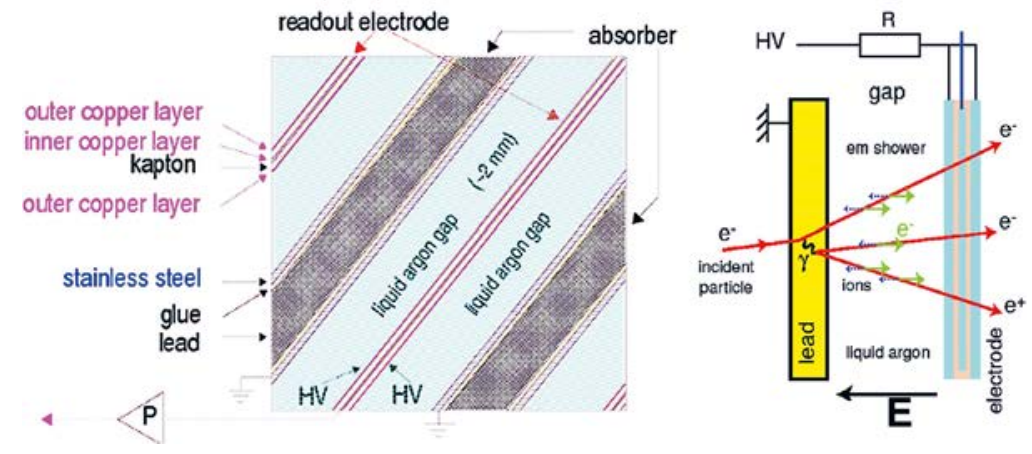

uniformity of response, adaptability to high segmentation, radiation hardness and reasonable cost. Indeed, the price of a litre of LAr compares favorably with the price of a bottle of beer. A disadvantage is the operation at cryogenic temperatures that requires cryostats and elaborate cryogenic systems.

\section{The ATLAS experiment at the LHC}

The LHC, the world's largest particle accelerator located at CERN, accelerates and collides protons at four interaction points with center-of-mass energies of $13 \mathrm{TeV}$. In these collisions, new particles are produced, most of which decay instantaneously into more stable particles. Figure 2 shows a transverse cross-section of one wedge of the ATLAS detector [3] consisting of many layers of particle detectors which are arranged cylindrically around the beam axis and the interaction point. All measurements combined contribute to the reconstruction of the full event, including short-lived particles which are created in the collision but decay instantaneously.

Electrons and photons shower in the electromagnetic LAr calorimeter, whereas hadrons produce more penetrating showers and are absorbed inside the hadronic calorimeter.

\section{The ATLAS LAr electromagnetic calorimeter and its role in the discovery of the Higgs boson}

The ATLAS electromagnetic calorimeter is a lead-LAr sampling calorimeter with accordion geometry [4] enabling a uniform acceptance without any gaps for services. The width of the active LAr gaps (Figure 1) is $2 \mathrm{~mm}$ in the central region, with a drift field of $10 \mathrm{kV} / \mathrm{cm}$. The energy resolution of this calorimeter is $\sigma_{E} / E \approx 1.5 \%$ for typical photon energies from Higgs decays. As shown in Figure 3, the calorimeter is segmented into four longitudinal read-out layers. The strip layer is finely segmented to distinguish between photons and neutral pions $\left(\pi^{0}\right)$ decaying into two nearby photons. The photon pointing measurement aligns the measurements from the first and the second layer and extrapolates the photon trajectory to the interaction region. Such additional information obtained due to segmentation played a decisive role in the Higgs boson discovery. $\triangle \mathrm{FIG}$. 1:

Left: Stack of absorbers, electrodes and LAr gaps as realized in a typical sampling calorimeter (ATLAS LAr calorimeter, see below) Right: Ionization charges produced by charged particles traversing a LAr gap. $\varangle$ Photo of the ATLAS barrel calorimeter and inner tracker. The LAr electromagnetic calorimeter is located in the visible inside the gold-colored front-end crates. aluminum cryostat 


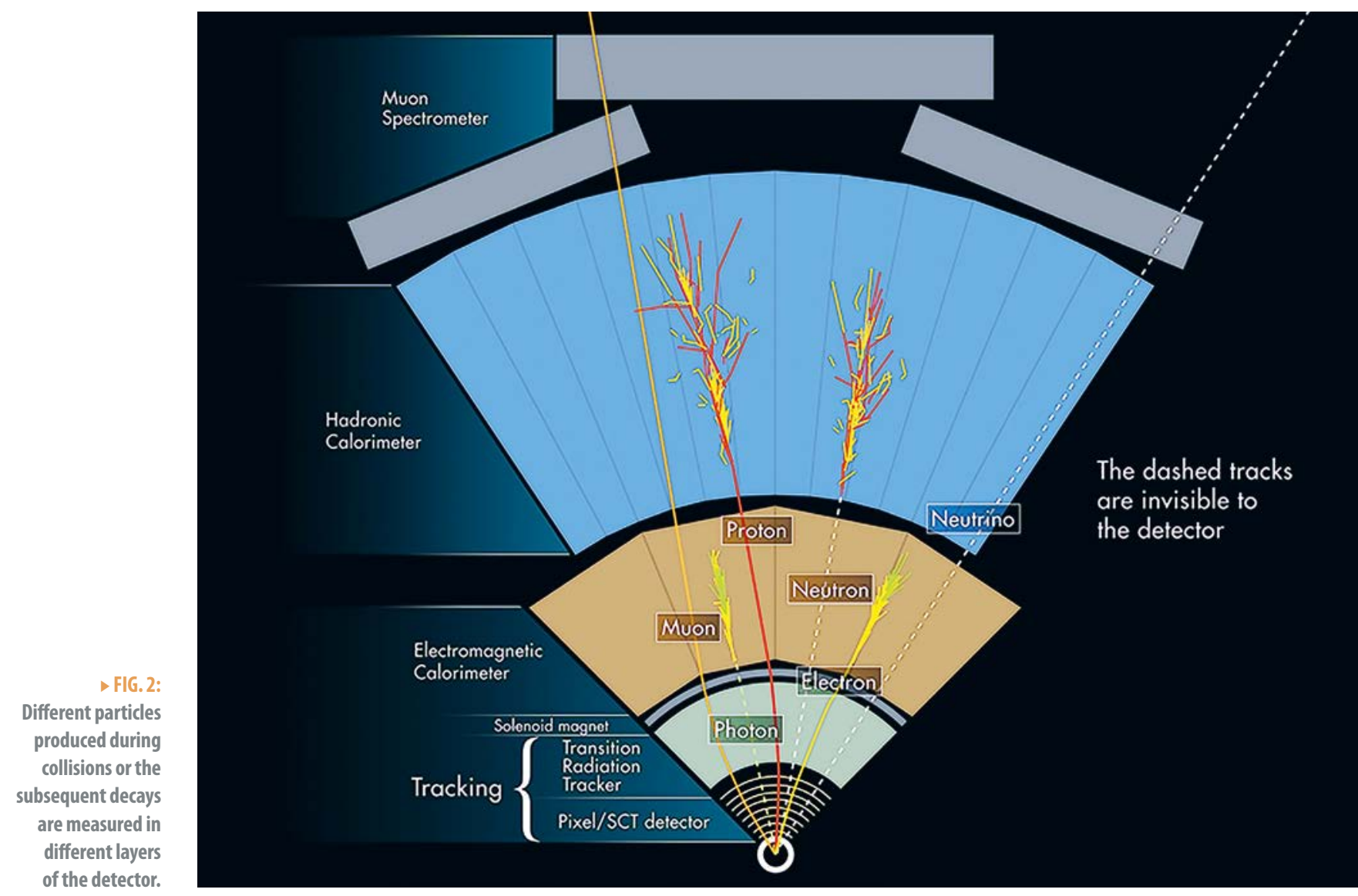

Already during the design of the LHC detectors, the Higgs decay into a pair of photons was considered to be one of the most promising signatures, and was chosen as a benchmark for the optimization of the LHC detectors, especially the electromagnetic calorimeters.

In July 2012, the discovery of the Higgs boson in the ATLAS and CMS experiments was indeed driven by the high significance of the two-photon decay $[5,6]$. The two detectors use complementary concepts of their electromagnetic calorimeters: instead of a sampling calorimeter, CMS opted for a homogeneous lead tungstate crystal calorimeter $\left(\mathrm{PbWO}_{4}\right)$ providing excellent energy resolution. The crystal calorimeter of CMS outperforms ATLAS in the photon energy resolution, whereas the segmented ATLAS

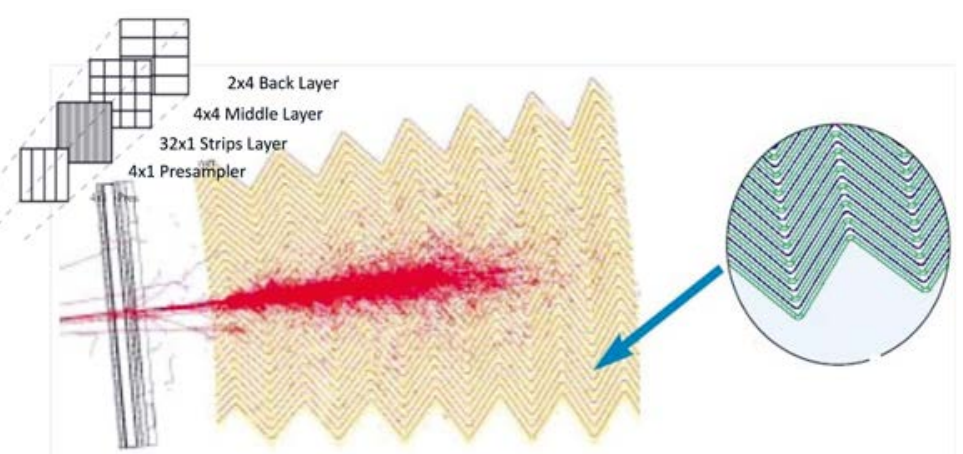

Particle flow optimizes the jet energy resolution by reconstructing each particle individually and by using the best measurement for each; charged particles measured in the tracker, photons and $\pi^{0}$ in the electromagnetic calorimeter, neutrons in the hadronic calorimeter.
LAr calorimeter compensates the worse resolution by photon pointing which helps to determine the correct primary vertex, crucial to calculate the transverse momentum and hence the invariant mass of the Higgs boson.

\section{Noble liquid calorimetry} in future collider experiments

The Future Circular Collider (FCC) is an ambitious project for an accelerator complex at CERN in the post-LHC era. An electron-positron collider, FCC-ee [7], is considered as a possible first step to measure precisely the Higgs properties and improve the measurement of key electro-weak parameters by several orders of magnitude. The tunnel length of $100 \mathrm{~km}$ is chosen to later house a $100 \mathrm{TeV}$ hadron circular collider, the FCC-hh [8].

FCC-ee

The intrinsic stability, uniformity, excellent linearity and energy resolution as well as its adaptability to high segmentation make noble liquid calorimeters a promising candidate for the next generation of lepton collider or "Higgs Factory" experiments. At the FCC-ee [7] Standard Model precision measurements at the $\mathrm{Z}$ pole will benefit from statistical uncertainties up to 300 times smaller than at LEP. Also, precision measurements of the Higgs properties will have to rely on an extremely well controlled systematic error which requires an excellent understanding of the detector and the event reconstruction. Highresolution calorimetry and an efficient particle-flow combination with the tracker measurement will be a prerequisite to achieve these ambitious physics goals. 


\section{FCC-hh}

Experiments at the FCC-hh with unprecedented luminosity will face an extreme radiation environment, where radiation-hard noble liquid calorimetry seems to be the most adapted, and possibly the only applicable technology. Up to 1000 simultaneous collisions will occur every $25 \mathrm{~ns}$ at the interaction points, producing extreme pile-up conditions potentially hiding interesting collisions of high momentum transfer. High segmentation of the electromagnetic calorimeter is a prerequisite to distinguish energy deposits from interesting collisions and to reject pile-up. It has been shown that noble-liquid calorimetry can be optimized in terms of segmentation to allow for 4D imaging, machine learning and - in combination with the tracker measurements - particle-flow reconstruction [9]. These techniques, together with excellent timing resolution, will be essential to reconstruct jets, reject pile-up and identify the proton collisions of interest.

\section{About the Author}

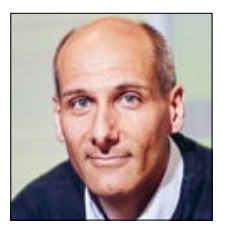

A native of Austria, Martin Aleksa is a senior physicist at CERN. He is a member of the ATLAS collaboration since the late 90 's and contributed significantly to the design, construction, operation and data analysis of the experiment. He led the ATLAS LAr calorimeter collaboration for several years. Recently he started to work on ATLAS HL-LHC upgrades and on the conceptual design of an FCC experiment with focus on calorimetry.

\section{References}

[1] Fabjan C.W., Fournier D. (2020) Calorimetry. In: Fabjan C., Schopper H. (eds) Particle Physics Reference Library. Springer, Cham. https://doi.org/10.1007/978-3-030-35318-6_6

[2] W.J. Willis, V. Radeka, Nucl. Instrum. Meth. 120 (1974) 221.

[3] ATLAS Collaboration, The ATLAS experiment at the CERN LHC, J. Instrum. 3, S08003 (2008)

[4] ATLAS Collaboration, Liquid Argon Calorimeter Technical Design Report, CERN/LHCC/96-041 (1996)

[5] ATLAS Collaboration, Phys. Lett. B 716 (2012)

[6] CMS Collaboration, Phys. Lett. B 716 (2012)

[7] A. Abada et al., Eur. Phys. J. Spec. Top. 228, 261 (2019). https://doi.org/10.1140/epjst/e2019-900045-4

[8] A. Abada et al., Eur. Phys. J. Spec. Top. 228, 755 (2019). https://doi:10.1140/epjst/e2019-900087-0

[9] M. Aleksa et al., CERN-FCC-PHYS-2019-0003, arXiv:1912.09962v1 [physics.ins-det] 20 Dec 2019

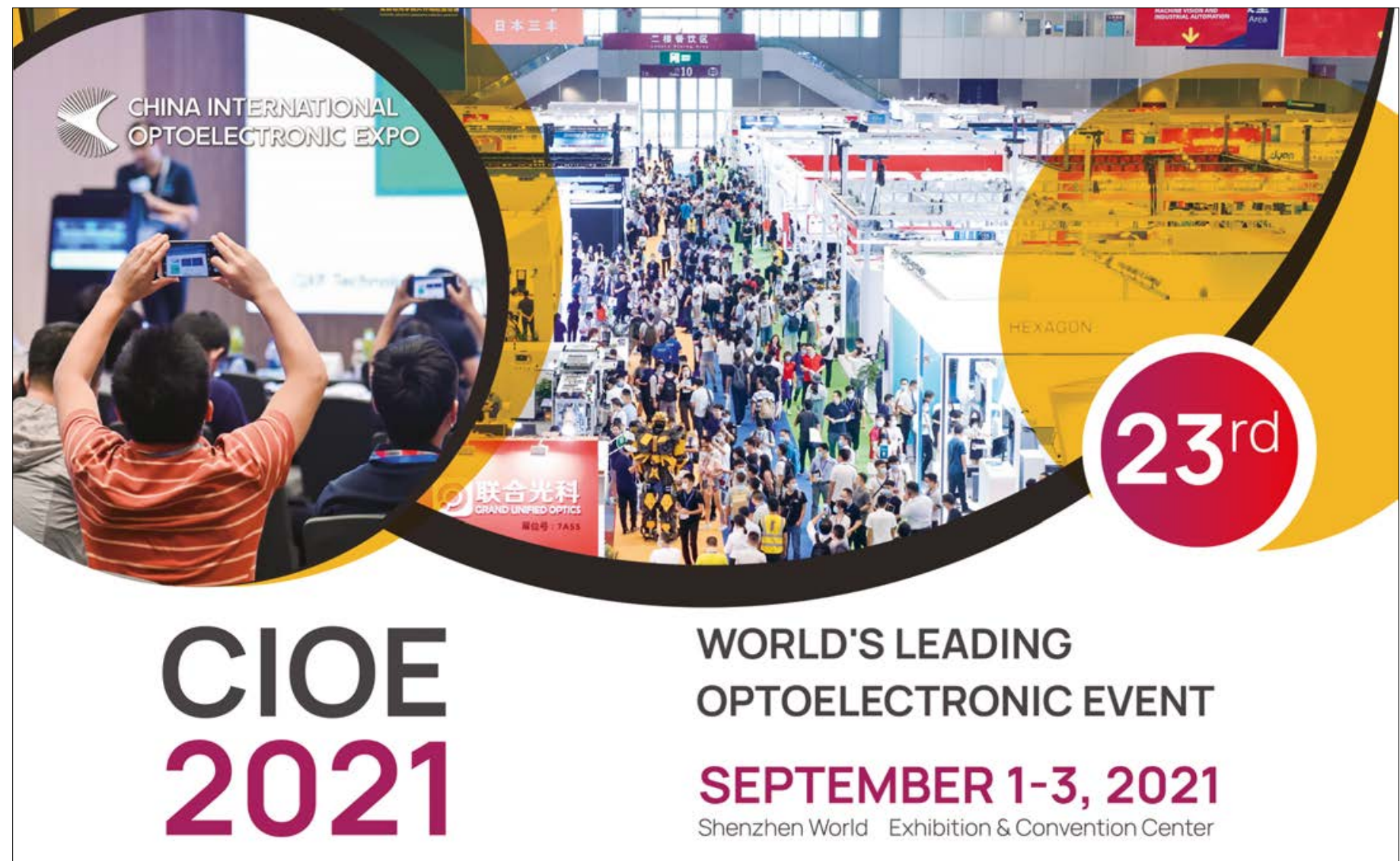

